\title{
Basic peculiarities of energy band spectra within generalized Kildal's model for semiconductors with one main axis
}

\author{
G.P. Chuiko, V.V. Martyniuk and V.K. Bazhenov \\ Kherson National Technical University, Department of General and Applied Physics \\ 24, Beryslavske shosse, 73008 Kherson, Ukraine, e-mail: chuiko@public.kherson.ua
}

\begin{abstract}
The analysis of the basic features of the generalized Kildal model had been presented for the semiconductors without the center of symmetry and with one main crystal axis. It had been proved, that the Kramers' degeneration survives only along and against of the direction of the main crystal axis and at point $\Gamma(\mathbf{k}=0)$ within this model under the condition of the absence of the center of symmetry, as rule. The Kramers' degeneration is possible everywhere, although only for the band of the heavy holes like within Kane's model, even under the previous condition, but only if casually takes place the special relation between parameters. The typical set of solutions within this model consists of one conductivity band $(\varepsilon \geq 0)$ and of three valence bands $(\varepsilon \leq 0)$. Each of them contains two spin subbands. The additional conductivity band would be possible in principle, but only under special condition $(\delta<0)$, which is the obligatory but not enough condition.
\end{abstract}

Keywords: energy bands, degeneration, Kramers, splitting, spin sub-bands.

Manuscript received 31.03.05, accepted for publications 18.05.05.

\section{Introduction}

A generalization of the known Kildal model had been reported [1] recently. This version describes the energy band spectra of semiconductors with one main axis as well as prior models [2, 3]. On the other hand, the new version is applicable not only to the crystals with the center of symmetry as [2, 3], but also to those, where such centers are missing. Moreover, there takes into consideration oneself and the lattice deformation along the main axis.

The Hamiltonian with its exact dispersion law had been presented in [1]. Authors reported that it had been possible to present the characteristic polynomial of the Hamiltonian as a product of two different polynomial factors. Both had the same degree. Indeed the dispersion law [1] looks like following with spherical coordinates $(k, \theta, \varphi)$ :

$\left(\Gamma(\varepsilon)-(P k)^{2}\left(f_{1}(\varepsilon) \sin ^{2}(\theta)+f_{2}(\varepsilon) \cos ^{2}(\theta)\right)\right)^{2}-$

$-(P k)^{2} f_{3}(\varepsilon)^{2} \sin ^{2}(\theta)=0$.

This characteristic polynomial has the quite evident decomposition into the product of two factors. Let us to explain the sense of symbols. There:

$$
\begin{aligned}
& \Gamma(\varepsilon)=\varepsilon\left\{\begin{array}{l}
\left(\varepsilon-\varepsilon_{g}\right)\left[(\varepsilon+2 \Delta / 3)(\varepsilon+\delta+\Delta / 3)-2 \Delta^{2} / 9 \eta^{2}\right]- \\
-d^{2}(\varepsilon+2 \Delta / 3)
\end{array}\right\}, \\
& f_{1}(\varepsilon)=P^{2}\left((\varepsilon+\Delta / 3)(\varepsilon+\delta+\Delta / 3)-\Delta^{2} / 9 \eta^{2}\right)
\end{aligned}
$$

$$
\begin{aligned}
& f_{2}(\varepsilon)=\eta^{-4} P^{2} \varepsilon(\varepsilon+2 \Delta / 3), \\
& f_{3}(\varepsilon)=\left(\frac{2 P}{3 \eta}\right) \varepsilon \Delta d .
\end{aligned}
$$

All these are polynomials as to the energy of carriers $-\varepsilon$. Thereto: $\varepsilon_{g}, \Delta, P$ are three well-known Kane’s parameters [3] (the energy gap, the spin-splitting parameter and the matrix element of the impulse). Further $\delta$ is the known parameter of the crystal field [2] and $d$ is another parameter of the crystal field, which describes the absence of the symmetry center [1]. Lastly $\eta$ is the scalar factor taking into account the deformation of the lattice [4]. The expressions (2 to 5) are equally correct both for semiconductors with $\varepsilon_{g}>0$ and for others with $\varepsilon_{g}<0$. Zero of energy ( $\varepsilon=0$ ) is located at the top of the band of heavy holes at any of both situations.

However, the paper [1] did not contain the deep analysis of the equation (1) as well as the investigation of the influence of its features as for the energy bands spectra. Only the few details could be as exclusion from this statement. They were visible in a figure showing the typical dependences of $\varepsilon(k)$ for these two directions. On the other hand, there had been presented only one concrete material (by the way with $\varepsilon_{g}<0$ ), and therefore it remained not lucid even as far as these details are general like other similar crystals.

The main aim of this paper is the search of mentioned basic peculiarities of the energy band spectra 
within the model [1], and this investigation should be leaning mostly against the detailed algebraic analysis of the expressions (1 to 5 ). It should provide the selection of most common features of the energy band structures for all the semiconductors described by this model.

\section{The analysis of the dispersion law}

Let us first present two factors of (1) as:

$$
\begin{aligned}
& P_{\alpha}(\varepsilon, \theta)=\Gamma(\varepsilon)-(P k)^{2}\left(f_{1}(\varepsilon) \sin ^{2}(\theta)+f_{2}(\varepsilon) \cos ^{2}(\theta)\right)+ \\
& +P k f_{3}(\varepsilon) \sin (\theta), \\
& P_{\beta}(\varepsilon, \theta)=\Gamma(\varepsilon)-(P k)^{2}\left(f_{1}(\varepsilon) \sin ^{2}(\theta)+f_{2}(\varepsilon) \cos ^{2}(\theta)\right)- \\
& -P k f_{3}(\varepsilon) \sin (\theta),
\end{aligned}
$$

Let us rewrite further these polynomials to their canonical (alias monic) forms as regard regard to the main variable ( $\varepsilon$ ) by using the expressions (2 to 5 ):

$$
\begin{aligned}
& P_{\alpha}(\varepsilon, \theta)=\varepsilon^{4}-\left(\varepsilon_{g}-\Delta-\delta\right) \varepsilon^{3}-\left(\left(\sin ^{2} \theta+\eta^{-4} \cos ^{2} \theta\right) \times\right. \\
& \times(P k)^{2}-\left(\frac{2}{3}\right) \eta^{-1} \Delta d \sin (\theta)(P k)+\left(\frac{2}{3}\right) \Delta d^{2}+ \\
& \left.+\left(\frac{2}{3}\right) \varepsilon_{g} \Delta\left(\delta+\frac{\Delta}{3}\left(1-\eta^{-2}\right)\right)\right) \varepsilon- \\
& -\frac{\Delta}{3}\left(\delta+\frac{\Delta}{3}\left(1-\eta^{-2}\right)\right) \sin ^{2} \theta(P k)^{2} \\
& P_{\beta}(\varepsilon, \theta)=\varepsilon^{4}-\left(\varepsilon_{g}-\Delta-\delta\right) \varepsilon^{3}-\left(\left(\sin ^{2} \theta+\right.\right. \\
& \left.+\eta^{-4} \cos ^{2} \theta\right)(P k)^{2}+\left(\frac{2}{3}\right) \eta^{-1} \Delta d \sin (\theta)(P k)+ \\
& \left.+\left(\frac{2}{3}\right) \Delta d^{2}+\left(\frac{2}{3}\right) \varepsilon_{g} \Delta\left(\delta+\frac{\Delta}{3}\left(1-\eta^{-2}\right)\right)\right) \varepsilon- \\
& -\frac{\Delta}{3}\left(\delta+\frac{\Delta}{3}\left(1-\eta^{-2}\right)\right) \sin ^{2} \theta(P k)^{2} .
\end{aligned}
$$

The companion matrixes corresponding to both polynomials are almost identical:

$$
H_{\alpha}=\left(\begin{array}{cccc}
0 & 0 & 0 & -a_{0} \\
1 & 0 & 0 & -a_{1 \alpha} \\
0 & 1 & 0 & -a_{2} \\
0 & 0 & 1 & -a_{3}
\end{array}\right) ; \quad H_{\beta}=\left(\begin{array}{cccc}
0 & 0 & 0 & -a_{0} \\
1 & 0 & 0 & -a_{1 \beta} \\
0 & 1 & 0 & -a_{2} \\
0 & 0 & 1 & -a_{3}
\end{array}\right) ;
$$

here $a_{j}$ are the coefficients at $\varepsilon^{j}$ in the polynomials $(8,9)(j=0,1,2,3)$ and the additional index (at $\left.a_{1 \alpha}, a_{1 \beta}\right)$ shows the only two different coefficients in (8), (9) and only two different elements in matrixes (10). Therefore, the Hamiltonian that corresponds to the characteristic polynomial (1), acquires the block-diagonal structure with two sub-matrixes (10):

$$
H=\left(\begin{array}{cc}
H_{\alpha} & 0 \\
0 & H_{\beta}
\end{array}\right) \text {. }
$$

Such a shape of Hamiltonian means that its space of the eigenvectors is divided onto the two invariant subspaces that are non-equivalent generally speaking [5].

Let us find the resultant of polynomials, $\operatorname{res}\left(P_{\alpha}, P_{\beta}\right)$ by using of the standard algebraic method [6]. The result may be written in the form:

$\operatorname{res}\left(P_{\alpha}, P_{\beta}\right)=-\left(\frac{256}{729}\right)\left(\left(\eta^{2}-1\right) \Delta+3 \eta^{2} \delta\right) \times$

$\times \Delta^{5} d^{4} \eta^{-6}(P k)^{6} \sin ^{6} \theta$.

Any two polynomials have a mutual root if and only if their resultant is equal to zgro [6, 7]. Here are below few physically reasonable conditions providing a zero of above-mentioned expression:

$k=0$,

$\sin \theta=0$

$d=0$.

Every one of them provides also the automatic execution of the following condition:

$a_{1 \alpha}=a_{1 \beta}$.

In addition, as it follows from (16):

$P_{\alpha}=P_{\beta}, \quad H_{\alpha}=H_{\beta}$.

The first two conditions (13) and (14) simply signify that each of the energy levels is always twice degenerated at the point $\Gamma(\mathbf{k}=0)$ firstly, and along and against the direction of the main crystal axis secondly. These two statements are independent of the presence or absence of the symmetry center in a semiconductor. All energy levels are twice degenerated for any set of $(k, \theta)$ at presence of the symmetry center thirdly. It follows from the condition (15). Validity of these assertions is independent of the magnitudes or the signs of other model parameters. Thereby they are faithful for all semiconductors described by this model.

Although the formal possibility to think about the other trivial conditions (for instance, like $P=0$ and so on) as it is following from (12), but it seems senseless physically. Nevertheless, there is one non-trivial condition making the expression (12) equal to zero, specially:

$\delta=\frac{\Delta\left(1-\eta^{2}\right)}{3 \eta^{2}}$.

This condition looks like a relation between parameters of the model and may be satisfied, or be almost satisfied, randomly or artificially. That is why the condition (17) has in principle another quality as compared to the above conditions. The condition (17) leads to another but an equivalent condition:

$a_{0}=0$. 
The polynomials (8), (9) and matrixes (10) are still different, but get now one mutual root: $\varepsilon=0$. Thus, this mutual root is twice degenerated, and thereto is independent of the module $(k)$ as well as of the direction $(\theta)$ of the wavevector. It signifies physically, that we deal with the infinitely narrow band of the heavy holes like the same within Kane's model [3]. This analogy does not surprise, if to note that the condition of applicability of the Kane's model (i.e., $\eta=1$ and $\delta=0$ ) converts immediately the expression (17) into the identity. Such a kind of degeneration should be acknowledged as parametric dependent and consequently casual by the nature. That is why it has another quality and origin in contrast with the aboveconsidered kinds of the genuine degeneration and even with same within Kane's model.

At the same time, the other roots of characteristic polynomials all are different despite (17) and thus are single. It follows from the resultant between shortened polynomials, obtained from $P_{\alpha}$ and $P_{\beta}$ after the dividing of both on the energy $\varepsilon$. This resultant is equal to:

$\operatorname{res}\left(\frac{P_{\alpha}}{\varepsilon}, \frac{P_{\beta}}{\varepsilon}\right)=-\left(\frac{64}{27}\right)\left(\frac{k P \Delta d \sin \theta}{\eta}\right)^{3}$.

The expression is not equal to zero obviously under the condition (17) and returns us to conditions (13 to 15 ) if we keep in mind their degeneration.

One more theorem of the algebra of polynomials gives warranty that all roots of a polynomial are different each to other (and thus are single). It would be true, if only a polynomial is mutually simple with own first derivative as for main variable [5]. The direct calculation within well-known Euclid's algorithm shows that both greatest common divisors of the polynomials (8), (9) as for their derivatives are trivial, and thereby are equal to 1 . Thus, all the roots of polynomials are single under the condition, of course, that neither of the above-mentioned conditions (13 to 17) is satisfied. Moreover, the roots are different not only "inside polynomials", but "between them", too.

What type of the degeneration we are keeping in mind? The time inversion operator $\hat{K}$, for instance, can convert polynomials one into another as it turns of a motion and a spin state into own opposites. So from $\hat{K} \theta=\theta+\pi$ and $\hat{K}(\sin \theta)=-\sin \theta$ follows, that $\hat{K} P_{\alpha}=P_{\beta}$ and vice versa $\hat{K} P_{\beta}=P_{\alpha}$. The subscripts $\alpha, \beta$ should be recognized consequently as the indicators of the different spin states that are degenerated or not degenerated in dependence on the satisfaction of the terms (13 to 17).

It can be proved doubtless by the transformation of the Hamiltonian matrix (11) with operator $\hat{K}$. Indeed:

$$
\hat{K}\left(\begin{array}{cc}
H_{\alpha} & 0 \\
0 & H_{\beta}
\end{array}\right) \hat{K}^{-1}=(-1) \cdot\left(\begin{array}{cc}
H_{\beta} & 0 \\
0 & H_{\alpha}
\end{array}\right)
$$

Here $\hat{K}=\sigma, \hat{C}=\left(\begin{array}{cc}0 & i \\ -i & 0\end{array}\right) \hat{C}$, and $\hat{C}$ is the operator of the complex conjugation, and also takes into account that both blocks (10) have only the real matrix elements. The time inversion operator just permutes the blocks of Hamiltonian and there any phase multiplier does not matter physically, firstly. The time inversion and Hamiltonian are not commutative operators, so the wellknown Kramers theorem about the double degeneration does not act under condition $H_{\alpha} \neq H_{\beta}$, secondly. Well, it means we deal presently with the Kramers degeneration, if opposite $H_{\alpha}=H_{\beta}$ [4].

The characteristic polynomials (8 and 9) have both the fourth degree. This means that their roots could be obtained in radicals. However, it would be very tiresome and scarcely usefully to review these bulky expressions. It seems more important to find the distribution of roots of these polynomials on signs, especially in an asymptotic limit: $k \rightarrow \infty$. The coefficients of both polynomials should be identical, if it is possible to neglect almost all elements, except for those which contain senior (leading) degrees of $k \rightarrow \infty$. Let us write their asymptotic expressions:

$$
\begin{aligned}
& a_{4}=1 ; \quad a_{3}=\Delta+\delta-\varepsilon_{g} ; \\
& a_{2}=-P^{2} k^{2} ; a_{1}=-\left(\frac{2 \Delta}{3}+\delta \sin (\theta)^{2}\right) P^{2} k^{2} ; \\
& a_{0}=-\left(\frac{\Delta \delta}{3}\right) \sin (\theta)^{2} P^{2} k^{2} .
\end{aligned}
$$

It is accepted in these expressions an insignificant simplifying $\eta \approx 1$ for the sake of greater simplicity and transparency. The amount of changes of signs in the sequence (21) determines the amount of positive roots of the proper polynomial $(N)$. This assertion is known as Cartesian rule of signs [7].

Would we want to make no assumption about parameters of (21), so the distribution of signs should adopt the kind as following a pseudo-vector: (+,?,-,?,?). There are only six variants of sign distributions turning out a substitution in place of every indefinite sign (?) of one of certain signs $( \pm)$. It is easily to check that they correspond to three possible values of $N=1,2,3$, in pairs. Well, such super-generalized approach is not too fruitful. All, what is allowed to say is that every polynomial has at least one negative root (i.e. a valence band).

Let us accept only one, but physically quite obvious assumption that $\Delta>0$. As a result, we can immediately write two different pseudo-vectors: $(+$, ?,-,-,-) if $\delta>0$, and (+,?,-,?,+), if $\delta<0$. Moreover, it is $N=1$ (if $\delta>0$ ) or $N=2$ (if $\delta<0$ ), independently of the variants of the any substitutions instead of indefinite signs. Thus each polynomial has at least one (if $\delta>0$ ) or even two (if $\delta<0$ ) positive roots, but no more. One of them is the conductivity band, whereas three negative roots of polynomial correspond to three valence bands, of course. Note that these assertions base just on one, 
physically evident assumption about sign of one parameter.

However, the possibility of two positive roots as the alternative to the typical conditions a bit washes out this analysis. The nature of the second positive root needs the additional study in this case, in dependence off other parameters of model. Thus, it as yet is few outside of the area of the presented investigation.

\section{Conclusions}

The Kramers degeneration survives only along and against of the direction of the main crystal axis and at point $\Gamma(\mathbf{k}=0)$ within generalized Kildal's model under the condition of the absence of the center of symmetry, as a rule.

The Kramers degeneration is possible everywhere, although only for the band of the heavy holes like those within Kane's model, even under the condition of pt.1, but only if casually takes place the special relation between parameters: $\delta=\frac{\Delta\left(1-\eta^{2}\right)}{3 \eta^{2}}$.

The typical set of solutions within this model consist of one conductivity band ( $\varepsilon \geq 0$ ) and of three valence bands $(\varepsilon \leq 0)$. Each of them contains two spin subbands. The additional conductivity band would be possible in principle, but only under special condition $(\delta<0)$, which is the obligatory but not enough condition.

\section{References}

1. G. Chuiko, N. Don, O. Dvornik, V. Ivchenko, A. Sergeev, Simple inverted band structure model for cadmium arsenide $\left(\mathrm{Cd}_{3} \mathrm{As}_{2}\right)$ // Moldavian Journal of the Physical Sciences, 2(1), p. 88-94 (2003).

2. H. Kildal, Band structure of $\mathrm{CdGeAs}_{2}$ near $\mathbf{k}=0$ // Phys. Rev., 10(12), p. 5082-5087 (1974).

3. E.O. Kane, Band structure of indium antimonide // J. Phys.Chem. Solids 1, p. 249-261 (1958).

4. G.L. Bier, G.E. Pikus, Symmetrya i deformatsionnye effekty w poluprovodnikach, Nauka, Moscow (1972) (in Russian).

5. V.V. Woyevodin, Y.A. Kuznetsov, Matricy i vychislenya, Nauka, Moscow (1984) (in Russian).

6. A.G. Kurosh, Kurs vysshey algebry, Nauka, Moscow (1968) (in Russian).

7. G.A. Korn, T.M. Korn. Mathematical Handbook for scientists and engineers, McGraw Hill Book Company, New York-San Francisco-TorontoLondon-Sydney (1968). 